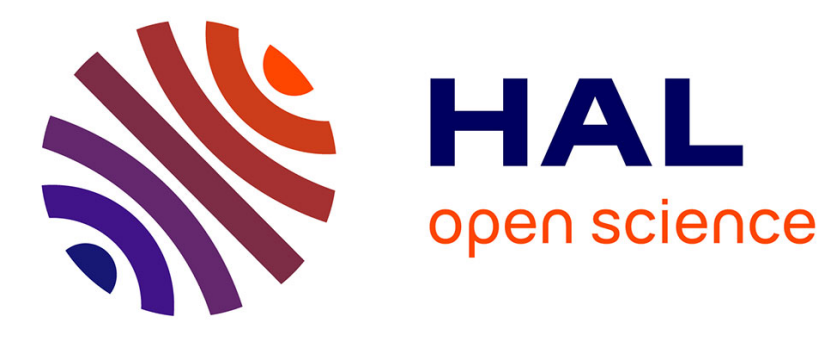

\title{
TV Interaction Beyond the Button Press
}

Regina Bernhaupt, Antoine Desnos, Michael Pirker, Daniel Schwaiger

\section{To cite this version:}

Regina Bernhaupt, Antoine Desnos, Michael Pirker, Daniel Schwaiger. TV Interaction Beyond the Button Press. 15th Human-Computer Interaction (INTERACT 2015), Sep 2015, Bamberg, Germany. pp.412-419, 10.1007/978-3-319-22668-2_31. hal-01599878

\section{HAL Id: hal-01599878 \\ https://hal.inria.fr/hal-01599878}

Submitted on 2 Oct 2017

HAL is a multi-disciplinary open access archive for the deposit and dissemination of scientific research documents, whether they are published or not. The documents may come from teaching and research institutions in France or abroad, or from public or private research centers.
L'archive ouverte pluridisciplinaire HAL, est destinée au dépôt et à la diffusion de documents scientifiques de niveau recherche, publiés ou non, émanant des établissements d'enseignement et de recherche français ou étrangers, des laboratoires publics ou privés. 


\title{
TV Interaction Beyond the Button Press \\ Exploring the Implications of Gesture, Pressure and Breath as Interaction Mechanisms for a TV User Interface
}

\author{
Regina Bernhaupt ${ }^{1}$, Antoine Desnos ${ }^{1}$, Michael Pirker ${ }^{2}$, Daniel Schwaiger ${ }^{2}$ \\ ${ }^{1}$ ICS team, IRIT, Université Paul Sabatier, Toulouse, France \\ \{regina.bernhaupt, antoine.desnos\}@irit.fr \\ ${ }^{2}$ user experience research, ruwido austria gmbh, Neumarkt, Austria \\ \{michael.pirker, daniel.schwaiger\}@ruwido.com
}

\begin{abstract}
In order to enhance users' interactions with TV user interfaces we developed a prototypical multimodal interaction mechanism that combines tilting, pressing and puffing as input modalities for a novel interface. The interaction mechanism has been evaluated in an exploratory user experience and usability study that used a within subjects design investigating tilt as input mechanism to navigate through the 3D interface compared to tilt combined with pressure and breath input. Results of this first exploratory study indicate that while this uncommon and unfamiliar way to interact with a novel TV user interface impacts usability scores which were below average compared to traditional remote controls, the user interface approach in combination with the new interaction modalities resulted in above-average scores for the user experience dimension of hedonic quality. The findings are subsequently reflected and implications of using alternative input modalities for TV user interfaces are discussed.
\end{abstract}

Keywords: Remote Control, Breath, 3D Position, Gesture, TV, User Interface, UI, User Experience, Usability

\section{Introduction}

Today TV user interfaces present more content and functions than ever before. Current offers enable users to watch hundreds of TV channels, provide an electronic program guide (EPG), and include a variety of apps or functions allowing to buy video on demand (VoD) movies and series, to record programs or to time-shift a program. To help users to interact with this multitude of services, the TV user interface has to provide an easy and intuitive menu structure as well as interaction mechanisms that can deal with this multitude of content and functionality, while at the same time ensuring to provide a good user experience (UX). While limitations of standard remote controls have been reported in detail (e.g. [5]), interacting with a TV is still associated with the use of a ordinary infra-red remote control by the majority of people, although also the remote control saw a variety of changes over the past 50 years, including the 
extension of number of buttons, possibilities for text entry, button reduction in conjunction with on-screen UIs, and the usage of modalities like touch or speech.

The general goal of our research is to investigate new forms and combinations of modalities to enhance TV user interfaces beyond standard grid-based structures and to find an interaction mechanism that is providing a novel and positive user experience. In this paper, we present results of a study that uses gesture as a possible means for playful interaction in the context of IPTV. Our research hypotheses were based on the idea that through the use of a playful approach with multimodal input (that is also used by e.g. gaming devices like the Nintendo Wii or Microsoft X-Box Kinect), a positive impact on the UX should be observable. Furthermore, we wanted to investigate the usability of gestures, especially accelerometer based gesture (tilt), as well as other modalities like pressure (press) and noise/voice input (puff) for TV interaction.

\section{Related Work}

\subsection{Gesture Interaction and Interactive TV}

Von Hardenberg and Bérard [3] discuss three types of requirements for a system using gesture interaction: the ability to detect or recognize gesture interaction, the identification (which type of object from a certain class is present - e.g. the recognition of certain movement and gestures), as well as the capacity to track gestures.

Gesture is widely accepted as a possible means of controlling devices due to its success in video game consoles like the Nintendo Wii and Microsoft Xbox Kinect. When gestures are applied in a TV environment, it offers the advantages that it does not require visual attention from the user on the remote control, but supports a completely blind usage, enabling the user to solely focus on the TV screen, and is reported to be well accepted by users [7,9]. Gesture interaction based on a Wii-mote has been investigated in the context of TV [1], replacing traditional remote controls. Gestures seem appropriate to enable eyes-free interaction, and to avoid the continuous problem of selecting buttons on a standard remote control, although typing on a physical remote control will remain faster than using gesture to control the TV [1]. Vatavu [11] proposed a set of commands for interacting with augmented TV environments using gesture with a Wii controller. It was observed that the majority of participants preferred buttons to motion gestures, especially for abstract tasks (e.g. mute or menu), and that purely gestural techniques tend to be slightly lower in terms of performance. Gesture was also used in combination with mobile phones and real 3D TV simulations to investigate new forms of control on the mobile phone screen [4], differing from the work reported in this paper as two screens were involved.

\subsection{Pressure, Deformation and Noise as Input}

Hoggan et al [6] dealt with the question whether squeezing is an effective input modality of mobile devices and if tactile feedback improves performance, as well as the effectiveness of squeezing as a manipulative interaction technique for use in mobile devices. Results for menu selection tasks show that squeezing is significantly 
faster than tilting, with and without tactile feedback, while both conditions facilitate successful interaction.

Not only voice, but also noise has been used to interact with user interfaces. Especially breath has been used by several researchers to implement interaction with interactive applications. Patel and Abowd [8] presented BLUI, a localized blowable user interface that allowed hands-free interaction via blowing at a laptop or computer screen to directly control certain interactive applications.

\section{Problem Description}

Our research goal and motivation was to investigate and get a deeper understanding of the usability and user experience when using accelerometer-based gesture (tilt) to navigate in a user interface that is not grid-based, but uses information representations in a pseudo 3D form of presentation. Based on recent positive reports from the general adoption of Wii-based interaction we wanted to investigate whether accelerometer-based gesture input results in the same Usability (measured with SUS questionnaire) and user experience (measured with AttrakDiff questionnaire) ratings than traditional TV interaction mechanisms (standard remote controls on standard IPTV UIs), as well as the implications on usability and user experience if the gesture input is enhanced with further input modalities (pressure, puffing). The comparison to traditional TV interaction mechanisms has been carried out by comparing the questionnaire scores to those from previous work in this field [10].

\section{The Prototypical System}

In the exploratory evaluation study, a prototypical user interface called Bubble UI and a remote control prototype described subsequently were used. The Bubble User Interface was designed with the goal to have an easy and intuitive way to navigate within large quantities of content, offering a novel and playful experience and improving the overall experience of the users. The UI represents the menus and content as a continuous and dynamic stream of information instead of static navigation and content elements. The representation of menus and content is carried out using dynamic round elements, which are visualized using the design metaphor of floating soap bubbles in the 3D space that can be directly manipulated in the synchronized user interface using the custom-tailored interaction mechanism. Further information on interaction, design rationale and further design aspects of the Bubble UI are discussed by the creators of the user interface in [2].

In order to complete our goal to offer a novel experience, we combined a set of analog inputs along with the GUI design. We chose Tilt, Press and Puff interaction as inputs because of both their analog nature and their close relation to the shapes and animations in the UI to ensure a consistent, novel and playful interaction ecosystem.

The remote control prototype which was used in the study was built using an Arduino Uno microcontroller board and sensors which were attached to an already existing remote control. 
The gesture and the pressure modalities were implemented using the Arduino components, while the microphone used for voice was already included in the remote control that was used (cf. Fig 2). The Arduino sensors we used in this case were a standard force resisting sensor (FSR) and the ADXL335 accelerometer. The sensors were cased nicely in the remote control.

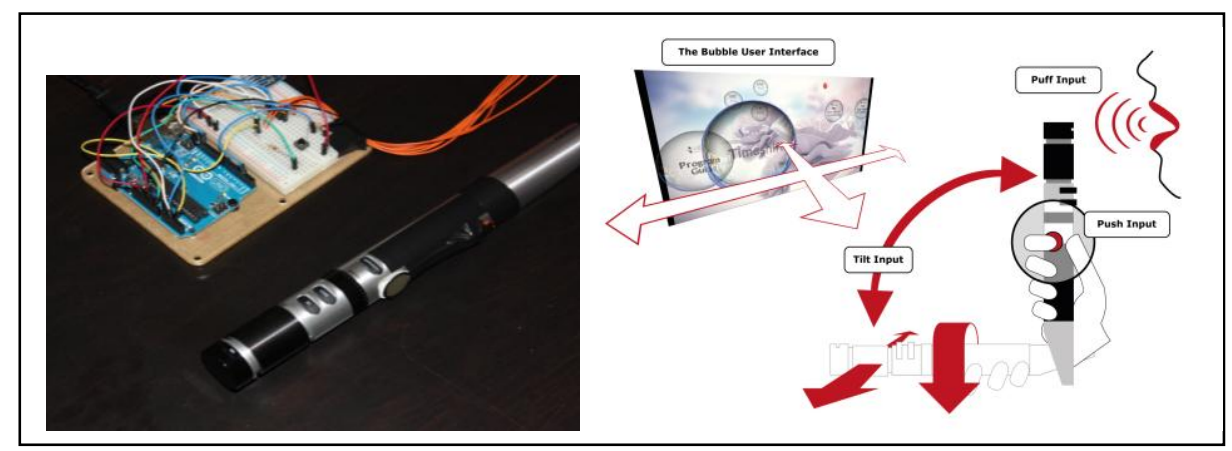

Fig. 2. Left image: The remote control prototype and the Arduino Uno Board; right image: Interaction with the Bubble UI: Possible movements were left/right and up/down.

We kept the Arduino board separated from the remote and hidden under a coffee table. The sensors were connected using one meter long cables. The accelerometer was glued inside the battery compartment to ensure that it will only move when the remote moves with the users hand. The FSR was attached to the side of the remote control and fixed in reach for left as well as right handed persons using insulating tape. The audio input from the built-in microphone controlled the puff input for accessing the main menu and the 'back' functionality. In the exploratory study, the navigation in the $3 \mathrm{D}$ space of the UI was carried out using the position-sensitive remote control prototype by tilting the remote control on two axes, forward, backward, left or right (see Figure 2). In one of the study conditions - the enhanced condition the prototype used a pressure-sensitive sensor that had to be pressed beyond a certain threshold for selecting an item (incl. visual feedback on force applied). The 'puff' functionality took over the metaphor of real soap bubbles and accessed the main menu (i.e. bubbles with menu items appeared on screen), or in the case of an already displayed menu hid the menu content (i.e. the user 'blew' the soap bubbles away).

\section{Exploratory Evaluation Study}

An experimental user study was conducted to investigate usability and user experience of the proposed user interface with accelerometer-based gesture input. The study followed a traditional usability study method including observation, interviews and domain-specific tasks to perform. Participants filled in the standard usability scale (SUS) questionnaire to measure usability, and the AttrakDiff questionnaire (www.attrakdiff.de) to measure the perceived user experience with the dimensions of pragmatic quality, hedonic quality and attractiveness. 
To limit the influence of possible learning effects and position effects the remote control order (i.e. the order of the modalities in the study), task order, age group and gender were counterbalanced.

Eight participants aged from 21 to 53 years were recruited for the study. All participants watch TV on a regular basis, had previous experiences with touch-screens and had already used position sensors in smart phones. Six participants also indicated familiarity with voice input. Observation was carried out using network-cameras and microphones. The user interface prototype was running on a small form factor computer, providing the UI and TV content in Full HD resolution on a flat screen TV set.

After a short pre-interview, every participant performed both experimental conditions - the basic accelerometer-based gesture interaction condition (BC) with traditional buttons for ' $\mathrm{OK}$ ' and 'Back', and the enhanced condition (EC) using pressure for 'OK' and puff for 'Back' functionality. For both remote controls (experimental conditions) participants had a short exploration phase followed by short questions on the first impression of system and interaction. Then participants performed three tasks where we measured task time, task success and task rating. Usability problems and errors were noted by the experimenter. After each remote control participants were asked to complete SUS and AttrakDiff questionnaire, followed by a short intermediate interviews and a final interview at the end of the study. The tasks for the study were chosen from a pool of IPTV tasks representing typical tasks for these systems (e.g. searching EPG for broadcasts, playing VOD movies, finding music in a library). Help was prepared if participants were stuck for a certain time period.

\section{$6 \quad$ Results}

Usability Problems. Most errors in interaction were related to precision problems with the position-sensitive navigation, including overshooting and problems finding the neutral position of the remote control. Participants were overlooking menu items in the UI and had problems with speed or accuracy for both types of interaction. On average, the enhanced condition performed faster for tasks with the EPG, while the base condition performed faster for the tasks with the music library, although no statistically significant differences was observed. Also completion rates for all tasks were investigated for differences between the enhanced condition and the base condition. Task completion overall was $73 \%$, but no significant difference in task completion for the two experimental conditions were observed. Furthermore, task times and ratings of perceived difficulty were investigated, but no statistical difference for the two conditions were observed. Tasks overall were perceived as difficult to complete.

Standard Usability Score (SUS). The novel interaction concept with the two tested conditions was assigned mediocre usability with room for improvement. The average rating for the enhanced condition was 52.8, while the base condition scored 50.3. There was no significant difference in SUS scores for study condition: $t(14)=0.22, p$ $=0.83$, nor for the age group: $\mathrm{t}(14)=0.83, \mathrm{p}=0.42$, or the position of the remote control in the study (first or second position): $\mathrm{t}(14)=0.22, \mathrm{p}=0.83$. 
User Experience - AttrakDiff questionnaire. The enhanced condition with gesture, voice and pressure achieved higher ratings for both HQ-I (identification) as well as HQ-S (stimulation), while the ratings for the pragmatic quality (PQ) were quite low, which is in line with findings from the SUS questionnaire and previous findings, e.g. for Touch-enabled remote controls [10]. The difference for PQ is not statistically significant $(\mathrm{t}(14)=0.02, \mathrm{p}=0.98)$. Although in terms of attractiveness both remote controls achieved an above-average rating (base condition: mean $=0.93, \mathrm{SD}=1.66$; enhanced condition: mean 1.00, SD=1.60), no statistically significant difference was observed $(\mathrm{t}(14)=-0.09, \mathrm{p}=0.93)$. The overall hedonic quality of the two remote controls, combining the concepts of hedonic quality - stimulation and hedonic quality identification, was rated high, with means between 1.33 and 1.55 on a scale from -3 to +3 for both remote controls. There was no significant difference in scores for hedonic quality $(\mathrm{t}(14)=-0.40, \mathrm{p}=0.69)$. Further statistical analysis took into account the gender and age group of the participants. While gender did not show significant differences in any AttrakDiff dimensions, the age group did. All but the stimulation dimension showed a significantly better rating by the younger age group (PQ: $t(8.09)=3.35$, $\mathrm{p}=0.01$; HQ-I: $\mathrm{t}(14)=3.13, \mathrm{p}=0.01$, ATT: $\mathrm{t}(14)=2.96, \mathrm{p}=0.01$, HQ: $\mathrm{t}(14)=2.72$, $\mathrm{p}=0.02)$, while the stimulation dimension only showed a trend $(\mathrm{t}(14)=2.09, \mathrm{p}=0.06)$.

First Contact and Retrospective UX Insights. After the free exploration phase, the participants were asked for their assessment of the interaction technology in conjunction with the UI after this short period. Results indicate that traditional TV systems are still preferred by the participants of the study. Although participants were unsure if they would find everything in the user interface and would not prefer it over their traditional TV systems, the results indicate that using the user interface with these remote controls is rather fun, as both of the remote controls were rated above average. After having completed the various tasks and filling in the questionnaires for each of the remote controls, the participants answered several questions in a short intermediate interview, asking for their impressions on the remote control, how natural it felt and how precise it was perceived. $47 \%$ of the participants overall stated that the navigation felt natural for them (33\% of answers of basic condition and $62.5 \%$ of answers for enhanced condition). Regarding the precision, $75 \%$ of participants ( $\mathrm{N}=8$ ) rated the precision of the enhanced condition as precise or rather precise, while the basic condition was rated as precise or rather precise only by $25 \%$ of participants.

Qualitative data from the interviews confirmed the other findings of the study. Participants had problems with and criticized the interaction modalities, while at the same time liking it and having fun. Especially the enhanced condition had many positive comments about the input modalities, although also problems and the need for improvements were reported. In the final interview, when asked about preferences for one of the remote controls, participants preferred the base condition remote control. In terms of usability people preferred to have buttons and the associated haptic feedback. Seven participants evaluated the base condition remote control as more reliable, while one person stated the remotes to be equally reliable. The main reason stated for this assessment was the buttons on the base condition remote control for six of the users. 


\subsection{Limitations}

In this paper, we investigated a prototypical multimodal interaction mechanism combining non-traditional input modalities for an UI that represents information in the form of soap bubbles. However, several limitations should be noted prior to the discussion of results. The rather low number of participants might have biased the results of the statistic analysis, but allowed us to quickly gather insights on usability and user experience issues as well as qualitative insights for further development. Another possible limitation is that the novel interaction modalities and novel type of UI might have had an impact on the hedonic user experience ratings, as novelty is a part of this dimension. One possible further limitation is that we did not have a baseline condition with a standard remote control, due to the fact that the UI was not designed to be controlled with standard input modalities. To overcome this limitation, we have used standardized and validated instruments like SUS and AttrakDiff that allow for comparison across systems and with previous work in the field [10].

\section{$7 \quad$ Discussion and Conclusion}

Our research goal and motivation was to investigate the usage of non-traditional input modalities for TV content, especially regarding implications of gesture input on user experience and usability scores. The perceived usability of the participants when interacting with the novel user interface with the non-traditional interaction modalities was clearly below average. These results are supported by below-average scores for the pragmatic quality dimension of the AttrakDiff questionnaire, as well as the comments of the participants in the interviews, and are in line with findings of Vatavu [11] on the performance of gestural techniques, as well as other work in the field on non-traditional interaction modalities [10]. This clearly indicates that the interaction modalities as well as the UI need further improved in terms of usability. The high perceived task difficulty for standard TV command and control tasks additionally indicates that the usability of the system and interaction technology needs further investigation and improvements in the future.

Suggestions for improvement of the prototypes mostly addressed interaction characteristics of the modalities: the position sensitive navigation, including the precision, the reaction to the user movements, the directness and the speed of deflection.

Concerning the user experience, our research hypotheses were that through using a playful approach with the multimodal input that is also used by e.g. gaming devices a positive impact on the user experience should be observed. Ratings in hedonic quality and attractiveness were high, indicating that although the usability, reflected in the pragmatic quality dimension, was below average, the novelty of the input modality and the user interface positively influenced UX.

Interestingly, the younger group rated the AttrakDiff questionnaire significantly better than the older age group. A touch-enabled remote control on a standard IPTV UI [10] scored similar to the study conditions presented in this paper, though, which might be interpreted that modalities beyond button press might improve UX, but not necessarily usability of interfaces in a standard TV command and control context. 
The study replicated previous findings [10] that the perceived user experience and perceived fun of an interaction is independent of its perceived usability, although repeated testing might be needed in order to verify the findings and account for the limitations of this exploratory study as described in the previous section. Evaluation in situ at people's homes for extended time periods might pose a good opportunity to gain more sophisticated insights why people accept or reject new modalities and further increase the reliability of the findings of this exploratory study in the future.

Nevertheless, the findings of the present study suggest that when designing for new interaction modalities and user interfaces for the IPTV domain, the key to increase user acceptance and adoption into the users' daily lives is not only by providing a high user experience in terms of attractiveness and hedonic quality, but also to ensure a high level of usability, especially for traditional command and control tasks. Thus, a take-away of the exploratory study presented in this paper is that although new interaction modalities' user experience benefits might stand out at first sight, ensuring to maintain a high level of usability that equals or exceeds the standard button interaction that users' are used to remains a crucial point when introducing new interaction modalities or concepts into the users' living rooms.

\section{$8 \quad$ References}

1. Bailly, G., Vo, D.-B., Lecolinet, E. and Guiard, Y. 2011. Gesture-Aware Remote Controls : Guidelines and Interaction Techniques. Proc. ICME 2001, 263 to 270.

2. Bernhaupt, R., Pirker, M., and Desnos, A. 2014. The bubble user interface: a tangible representation of information to enhance the user experience in IPTV systems. Proceedings DIS Companion '14, 85-88. ACM, New York.

3. von Hardenberg, C. and Bérard, F. 2001. Bare-hand human-computer interaction. In Proc. PUI '01, vol. 15, 1-8. ACM.

4. Halvey, M., Hannah, D., Wilson, G., and Brewster, S.A. 2012. Investigating gesture and pressure interaction with a 3d display. In Proc. MMM 12, 395 - 405. Springer.

5. Hess, J., Wan, L., Pipek, V., \& Kuestermann, G. 2011. Using paper and pen to control home-IT: lessons learned by hands-on experience. Proc. EuroITV '11, 203 - 212.

6. Hoggan, E., Trendafilov, D., Ahmaniemi, T., and Raisamo, R. 2011. Squeeze vs. tilt: a comparative study using continuous tactile feedback. In Ext. Abstr. CHI '11. ACM.

7. Kela, J., Korpipo, P., Marvi, J., Kallio, S., Savino, G., Jozzo, L. and Di Marca, K. 2006. Accelerometer-based gesture control for a design environment. Personal Ubiquitous Comput. 10, 5 (July 2006), pp. 285 to 299.

8. Patel, S.N., and Abowd, G.D. 2007. Blui: low-cost localized blowable user interfaces. In Proc. UIST '07.

9. Pelling, C., Sko, T. and Gardner. H. J. 2009. Be careful how you point that thing: Wiimote aiming for large displays. Proceedings OZCHI '09. pp. 397 to 400.

10. Pirker, M., Bernhaupt, R. and Mirlacher, T. 2010. Investigating usability and user experience as possible entry barriers for touch interaction in the living room. Proceedings EuroITV '10. pp. 145 to 154.

11. Vatavu, R-D. 2013. There's a world outside your TV: exploring interactions beyond the physical TV screen. In Proc. EuroITV '13, pp. 143-152. ACM New York. 